Bull. Mater. Sci., Vol. 16, No. 3, June 1993, pp. 171-175. OPrinted in India.

\title{
Growth of hexagonal selenium crystals: Observation of new growth morphologies
}

\author{
N V CHANDRA SHEKAR, K GOVINDA RAJAN, S RAJAGOPALAN* \\ and MOHAMMAD YOUSUF \\ Materials Science Division, Indira Gandhi Centre for Atomic Research, Kalpakkam 603 102, \\ India \\ * National Evening College, Thiruchirapalli 620001, India \\ MS received 21 September 1992; revised 6 March 1993
}

\begin{abstract}
In this communication, we report the single crystal growth of selenium by the solution method. We have observed a new mesh-like growth pattern in hexagonal selenium for the first time. Crystals having hexagonal morphology are packed together like hay stick bunches.
\end{abstract}

Keywords. Selenium; crystal growth; growth pattern; crystal morphology.

\section{Introduction}

Selenium is a very interesting and peculiar substance. In the liquid state it has properties intermediate between polymeric and metallic liquids. Such properties cause difficulties for crystal growth from melt. Recently Bisault et al (1991) and Ryschenkow and Faivre (1988) studied the nucleation, kinetics and modes of crystallization of liquid selenium. Similar studies on solution growth of selenium are rare and do not contain all desirable experimental information (Kolb 1969; Miyamoto 1977; Lucovsky 1979; Cooper and Westbury 1979).

We undertook the preparation of single crystals of monoclinic (m-Se) and hexagonal (h-Se) selenium, the two kinds of solid modifications of selenium, by solution growth techniques. These methods yield sufficient crystals for basic studies (Abdullayev et al 1969; Iizima et al 1969; Miyamoto 1977). We obtained monoclinic crystals of good quality and their external morphology matched well with features reported earlier (Abdullayev et al 1969; lizima 1969; Miyamoto 1977). Hexagonal crystals showed certain new growth patterns and external morphology. In this paper we report the preparation of $\mathrm{h}$-Se crystals and our observations on various growth morphologies.

\section{Materials and methods}

The starting material was $99.999 \%$ pure selenium obtained from M/s Hoboken, Belgium. The starting material was in the form of small spherical lumps. Few mg of the element was powdered and analysed by $\mathrm{X}$-ray diffraction technique. The diffractogram showed a clear broad peak and some sharp peaks, as depicted in figure 1 . The broad halo peak showed that a large fraction was in the amorphous state. The presence of hexagonal phase was clearly discernible. Monoclinic phase could not be detected.

The preparation of hexagonal crystals was attempted by the solution growth technique. It is known that selenium dissolves well in dilute aqueous solution of sodium sulphide $\mathrm{Na}_{2} \mathrm{~S}$. Therefore one normality aqueous solution of $\mathrm{Na}_{2} \mathrm{~S}$ was taken as the 


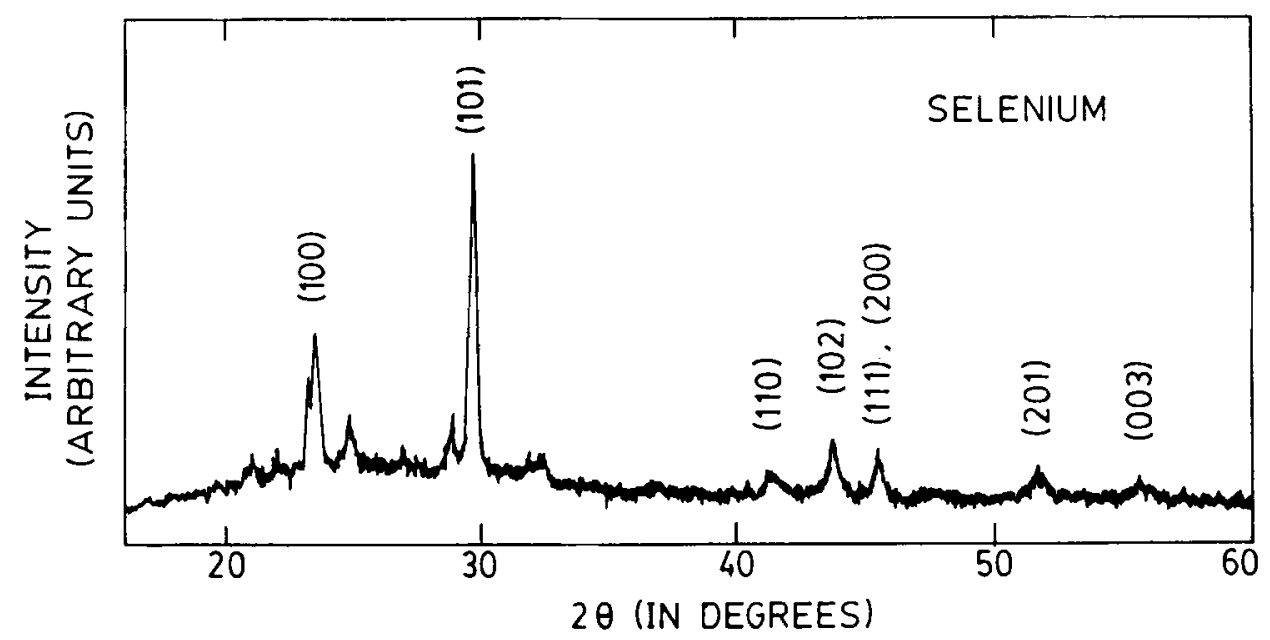

Figure 1. Powder X-ray diffractogram of pure selenium obtained from $\mathrm{M} / \mathrm{s}$ Hoboken, Belgium. The broad halo appearing between 20 of $20^{\circ}$ and $40^{\circ}$ is due to a-Se. The indices for the peaks are for $h$-Se. No trace of $m$-Se could be seen in XRD.

growth medium. Powdered selenium was dissolved in $1 \mathrm{~N} \mathrm{Na}_{2} \mathrm{~S}$ until the solution was saturated. The blood red-coloured, opaque solution was filtered and $30 \mathrm{ml}$ was taken for each run. The growth apparatus was simple and consisted of a temperature controller (Indotherm 401 D), an electric bunsen and a chromel-alumel thermocouple and was located in a disturbance-free area. Care was taken to adequately shield the beaker from fluctuations in the ambient temperature.

The growth was initiated by lowering the temperature of the solution from $50^{\circ} \mathrm{C}$ to $25^{\circ} \mathrm{C}$ at $1{ }^{\circ} \mathrm{C}$ per day. After the completion of the experiment the beaker and its contents were analysed.

\section{Results and conclusion}

It was found that inside the beaker a small fraction of liquid and white deposit was still left over at the bottom. There was a circular black deposit slightly above the bottom of the beaker. The deposit was very fragile. This was carefully dislodged from the beaker and taken for observation under a microscope.

Microscopic observation at a low magnification showed extensive mesh structure. There appeared at some places droplets of liquid residue. Closer observation revealed a thin layer of white-coloured deposit on top of the selenium surface. We presumed that the deposit was $\mathrm{Na}_{2} \mathrm{~S}$ from simple logic. The deposit was then carefully removed by washing with distilled water. This had to be done slowly and carefully since the selenium deposit had the tendency of breaking up into smaller bits in water.

The deposit was next observed at larger magnifications $(\times 160)$ and the criss-crossing mesh structure present extensively was clearly visible (figure 2 ). At some other regions structures similar to haysticks were observed. These were bunched together and each bunch contained several sticks. It is important to note that these structures were observed on the upper portion of the deposit, the lower portion being relatively smooth and featureless. The haystick structure is shown in figure 3 . The length of a single stick was around $300 \mu$ giving rise to a growth rate of $8 \times 10^{-9} \mathrm{~m} / \mathrm{min}$. 


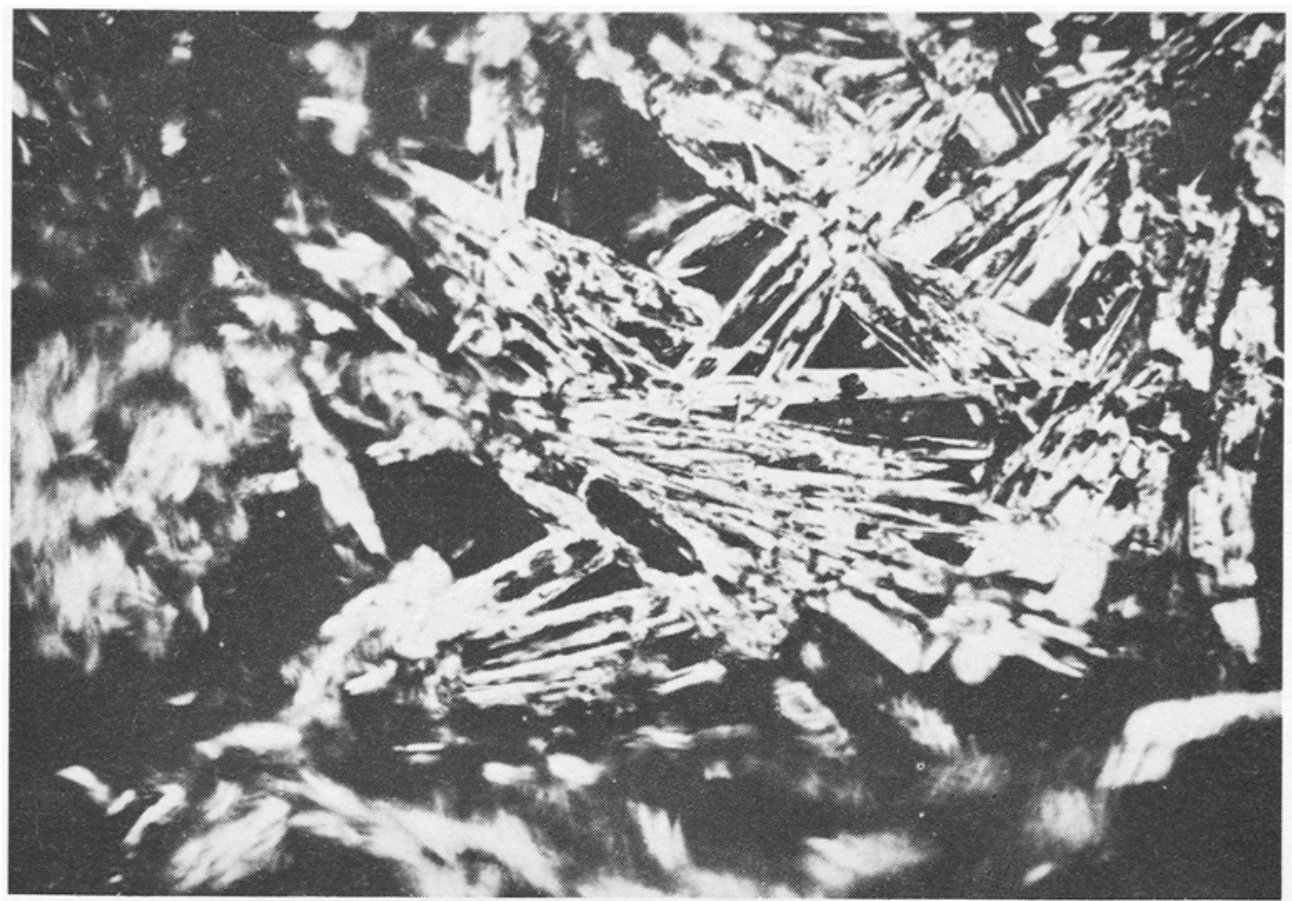

Figure 2. Regions of deposits showing meshy criss-cross structural pattern $(\times 240)$.

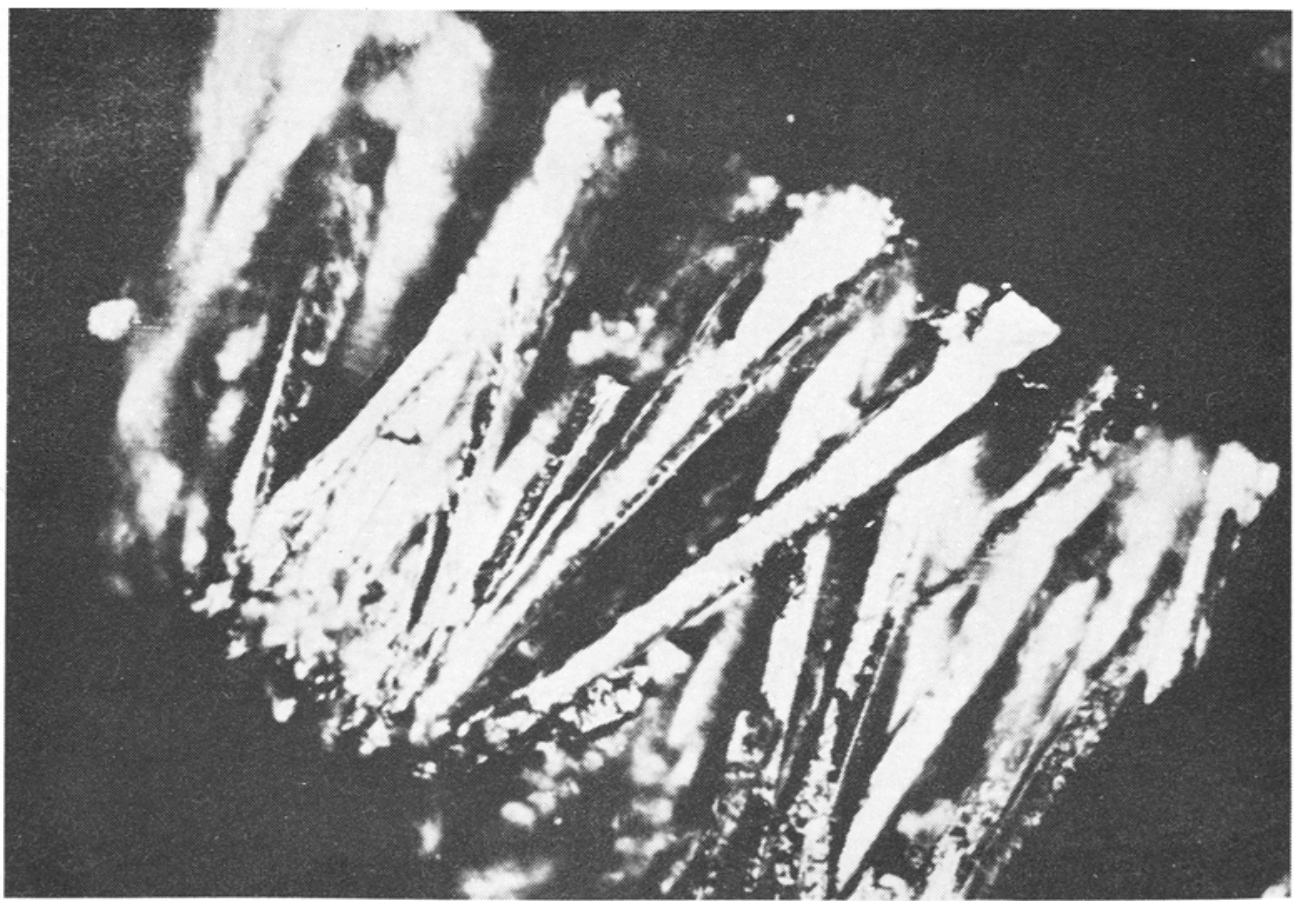

Figure 3. Haystick bunch morphology. Each stick is a hexagonal crystal of selenium $(\times 240)$. 


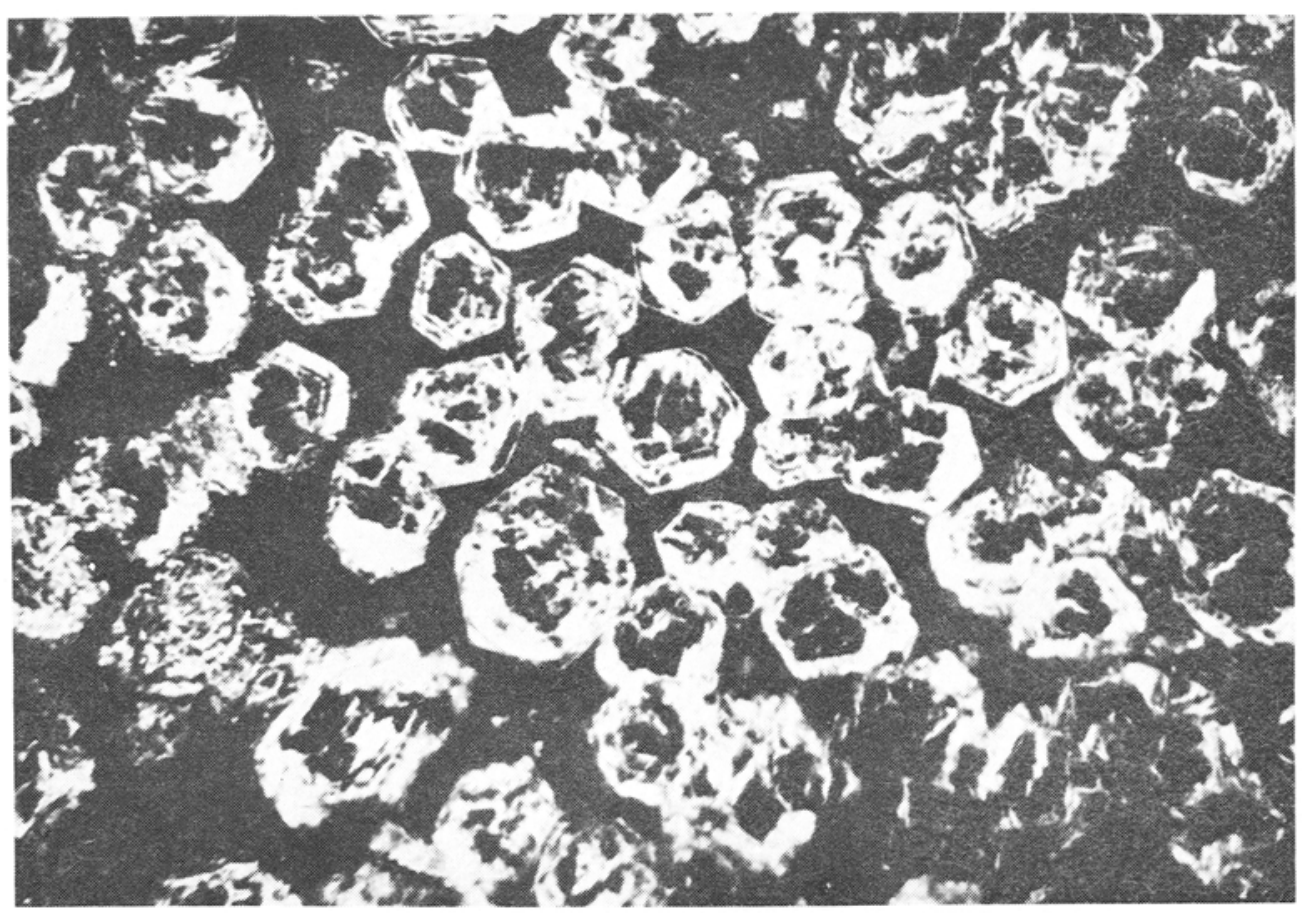

Figure 4. Cross-sections of haystick bunch showing hexagonal pattern, $(\times 240)$.

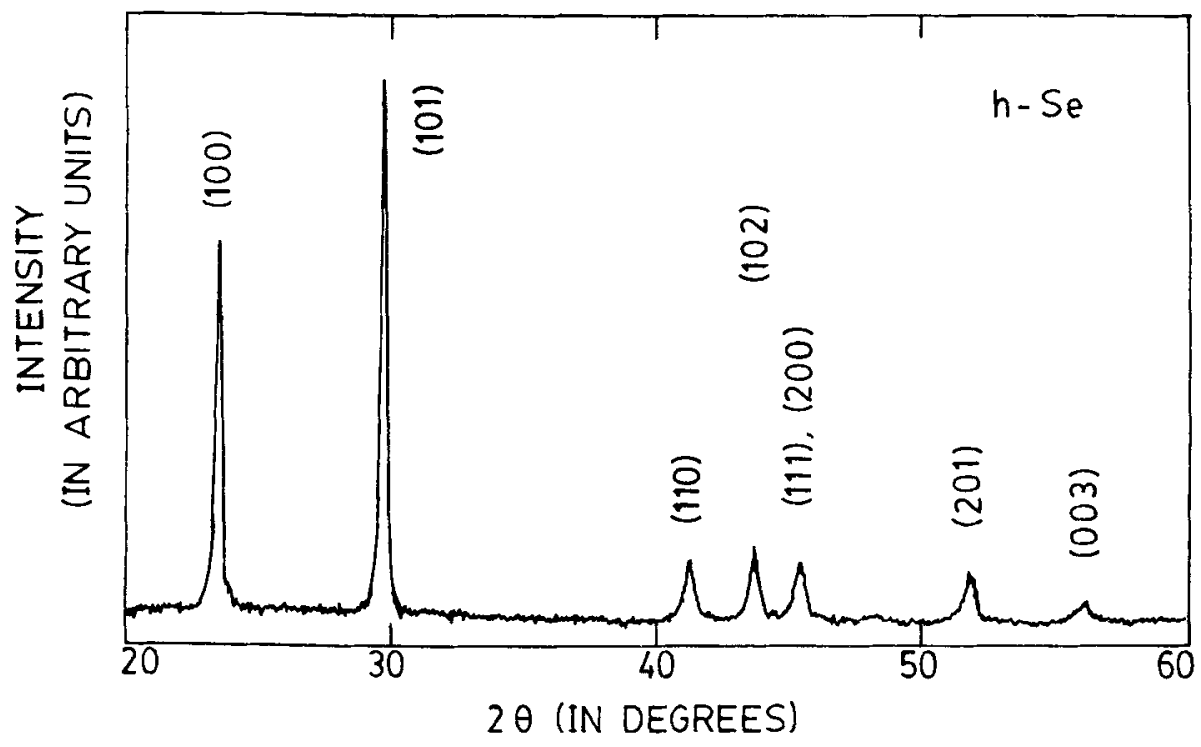

Figure 5. Powder X-ray diffractogram of powdered hexagona! crystals. All the peaks can be ascribed to the h-Se phase. 
Miyamoto (1977) reported similar needle-like crystals in another method of preparation and characterized them to be the new type of hexagonal crystals composed of $\mathrm{Se}_{6}$ rings. The outer surface of the observed haysticks had well-defined edges and a cross-sectional view is shown in figure 4 . The figure shows beautiful hexagonal patterns indicating clearly that these are good hexagonal single crystals. Some of these haysticks were carefully plucked from a sprout, powdered and X-ray diffraction pattern was carried out which confirmed their hexagonal form (figure 5).

A comparison between the melt growth and solution growth shows a case analogous to very low supercooled melt. For low supercooled melts, although the growth rate is very low, the yield is good as reported by Ryschenkow and Faivre (1988).

To conclude, we have reported some new and interesting morphology and growth patterns observed during single crystal growth of hexagonal selenium. The growth from sulphide solution yields mostly hexagonal crystals with very good yield. The growth morphology and patterns were reproducible. The size of the crystals can be increased by improving upon the method.

\section{Acknowledgement}

The authors thank Shri M Sekar and Shri L M Sundaram for their assistance during experiments. The authors thank Dr J Janaki for her help in obtaining X-ray diffractograms. Thanks are also due to Dr Kanwar Krishan for constant encouragement.

\section{References}

Abdullayev G B, Asadov Y G and Mamedov K P 1969 The physics of selenium and tellurium, (eds) W Charles Cooper, (Oxford: Pergamon Press) 103

Bisault J, Ryschenkow G and Faivre G 1991 J. Cryst. Growth 110889

Charles Cooper W and Westbury R A 1979 in Selenium (eds) A Zingaro Ralph and W Charles Cooper (New York: Van Nostrand Reinhold company) Chap 3, p. 87

Iizima S, Taynai J and Nicolet M-A 1969 The physics of selenium and tellurium. (ed.) W Charles Cooper (Oxford: Pergamon Press) p. 199

Kolb E D 1969 The physics of selenium and tellurium, (ed.) W Charles Cooper (Oxford: Pergamon Press) p. 155

Lucovsky G 1979 The physics of selenium and tellurium, (eds) E Gerlack and P Grosse (Berlin: SpringerVerlag) Part VII, p. 178

Miyamoto Y 1977 Jap. J. Appl. Phys. 162257

Ryschenkow G and Faivre G 1988 J. Cryst. Growth 87221 